\title{
BMJ Open Transcutaneous electrical acupoint stimulation (TEAS) for cancer-related fatigue: study protocol for a systematic review and meta-analysis
}

Yiwei Zeng (1) , ${ }^{1}$ Jialin Xia, ${ }^{2}$ Zhihan Chen (D) , ${ }^{1}$ Xiaoping Tian, ${ }^{3}$ Yulan $\mathrm{Ren}^{3}$

To cite: Zeng Y, Xia J, Chen Z, et al. Transcutaneous electrical acupoint stimulation (TEAS) for cancer-related fatigue: study protocol for a systematic review and meta-analysis. BMJ Open 2021:11:e049318. doi:10.1136/ bmjopen-2021-049318

- Prepublication history for this paper is available online. To view these files, please visit the journal online (http://dx.doi. org/10.1136/bmjopen-2021049318).

Received 25 January 2021 Accepted 12 October 2021
D) Check for updates

(c) Author(s) (or their employer(s)) 2021. Re-use permitted under CC BY-NC. No commercial re-use. See rights and permissions. Published by BMJ.

${ }^{1}$ School of Acupuncture and Tuina, Chengdu University of Traditional Chinese Medicine, Chengdu, China

${ }^{2}$ School of Nursing, Chengdu University of Traditional Chinese Medicine, Chengdu, China

${ }^{3}$ School of Chinese Classics, Chengdu University of Traditional Chinese Medicine, Chengdu, China

Correspondence to Professor Yulan Ren; renxg2468@163.com

\section{ABSTRACT}

Introduction Cancer-related fatigue (CRF) is a prevalent symptom in cancer survivors. Transcutaneous electrical acupoint stimulation (TEAS) has been reported as a promising therapy for CRF. This protocol is proposed for a systematic review that aims to assess the efficacy and safety of TEAS for CRF.

Methods and analysis Cochrane Central Register of Controlled Trials, PubMed, Medline, Embase, Chinese National Knowledge Infrastructure, VIP, Wanfang database, Chinese Biomedical Literature Database, Chinese Clinical Trial Registry System, ClinicalTrials.gov and WHO International Clinical Trial Registry Platform will be searched from inception to 31 January 2021 without language limitations. The eligible randomised controlled trials will be included. The primary outcomes include changes in the revised Piper fatigue scale, the Brief fatigue inventory, the Multidimensional fatigue inventory and the Functional assessment of chronic illness therapy fatigue. The secondary outcomes are the quality-of-life measurement index, the Hamilton anxiety scale, the Hamilton depression scale and adverse events. The selection of studies, data extraction and assessment of risk of bias will be conducted independently by two reviewers. Data synthesis will be performed using RevMan V.5.4.1. The quality of evidence will be evaluated with the Grading of Recommendations, Assessment, Development and Evaluation system. This study will strictly adhere to the Preferred Reporting Items for Systematic Review and Meta-Analysis guidelines.

Ethics and dissemination Ethical approval is not required as this is a systematic review and meta-analysis based on previously published studies involving no private information of patients. The results of this study will be disseminated in a peer-reviewed journal.

PROSPERO registration number CRD42020220282.

\section{INTRODUCTION}

Cancer-related fatigue (CRF), a common symptom in cancer survivors, is defined as a distressing, persistent, and subjective sense of tiredness or exhaustion that cannot be alleviated by sleep or rest. ${ }^{1}$ It is almost universal in those patients receiving anticancer treatments and affects nearly $65 \%$ of cancer survivors. $^{2-4}$ Approximately $62 \%-85 \%$ of patients

\section{Strengths and limitations of this study}

To the best of our knowledge, this study will be the first systematic review and meta-analysis to evaluate the efficacy and safety of transcutaneous electrical acupoint stimulation (TEAS) for cancer-related fatigue (CRF).

- The study will review quantitative data systematically from multiple databases to assess the efficacy and safety of TEAS for patients with CRF.

- This study follows the Preferred Reporting Items for Systematic Review and Meta-Analysis Protocols guidelines.

- Potential poor methodological quality, publication bias and small sample size of the included studies may be the limitations of the study.

with cancer who undergo active treatments experience $\mathrm{CRF}^{5}{ }^{5} \mathrm{CRF}$ is not just an isolated symptom, but associates with anxiety, depression and insomnia. ${ }^{6}$ It is a multifactorial condition involving anaemia, inflammationmediated changes of cytokines, cellular immunity dysregulation and oxidativestress-induced striated muscle dysfunction mediated by cancer or chemotherapeutic agents. $^{7-9}$ It inflicts a negative impact on patients' quality of daily life and may cause treatment discontinuation and survival reduction. ${ }^{810}$ However, it has often been underestimated, underdiagnosed and insufficiently treated. ${ }^{11}$ Though both pharmacological and non-pharmacological interventions have been applied in clinical management, a metaanalysis has shown that compared with nonpharmaceutical therapy, the efficacy of drugs on CRF is inferior with an increased risk of side effects. ${ }^{12} 13$ The gold standard for CRF management is still unavailable. ${ }^{14}$ Hence, an effective and safe treatment option remains an urgent need for patients with CRF.

Traditional Chinese medicine (TCM) has been widely used among cancer survivors in 
China and gradually accepted worldwide by its efficacy in recent years. ${ }^{15}$ As an integral part of TCM, acupuncture is being adopted by patients with cancer for a wide range of cancer-related symptoms, and some clinical trials have shown that acupuncture can provide clinical benefits for patients with CRF. ${ }^{16}{ }^{17}$ Transcutaneous electrical acupoint stimulation (TEAS) combines transcutaneous electrical nerve stimulation with acupoint stimulation and is a noninvasive alternative to acupuncture. Under the guidance of meridian theory, this technique stimulates acupoints on the surface with low-voltage pulses close to the body's bioelectricity and has been reported to relieve the varieties of cancer-related symptoms, including fatigue, immunosuppression and bone marrow suppression. ${ }^{18-21}$ In addition, compared with the traditional manual acupuncture that requires qualified acupuncturists or TCM clinicians to perform, TEAS can be implemented by nursing staff or patients themselves after training making it more accessible. ${ }^{22}$ Moreover, this non-invasive therapeutic approach is pain-free and more acceptable for patients with needle phobia. $^{23}$

In recent years, an increasing body of clinical trials has been carried out to evaluate the efficacy and safety of TEAS on patients with CRF, and the results have indicated it might be a promising therapeutic intervention. However, currently no systematic review has been reported to assess the clinical evidence. This study will include and systematically synthesise the eligible randomised controlled trials (RCTs) without language restrictions. To the best of our knowledge, this meta-analysis is the first attempt to assess the available evidence of TEAS for the treatment of CRF. Hopefully, this study may yield helpful information for the people concerned.

\section{Objective}

This systematic review aims to critically assess the efficacy and safety of TEAS for CRF.

\section{METHODS}

The study protocol will follow the Cochrane Handbook for Preferred Reporting Items for Systematic Reviews and MetaAnalyses Protocol statement guidelines (PRISMA-P). ${ }^{24} 25$

\section{Criteria for including studies for this review \\ Types of studies \\ We will only include randomised controlled trials (RCTs) investigating the efficacy and safety of TEAS on CRF. Cross-over trials and quasi-randomised trials will be excluded.}

\section{Types of participants}

We will include patients with CRF of any age or sex who have been diagnosed by any recognised diagnostic criteria (eg, the NCCN Clinical Practice Guidelines in Oncology (NCCN Guidelines) for cancer-related fatigue) or based on the vital characteristics of CRF (eg, a distressing, persistent and subjective sense of tiredness or exhaustion that could not be alleviated by sleep or rest). Participants with fatigue caused by other diseases will be excluded, such as hepatitis, anaemia and hypothyroidism.

\section{Types of interventions}

We will include RCTs that use TEAS with or without conventional medicine and exclude other invasive or noninvasive acupoint stimulation methods, such as acupuncture, laser stimulation, moxibustion and acupressure. No limitations will be placed on the duration of treatment.

\section{Types of comparator(s)/control}

Control interventions will be wait-list control, TEAS on corresponding non-acupoints, other methods of acupoint stimulation (eg, acupuncture, moxibustion and acupressure), and the same conventional anticancer drugs as the interventional group.

We will also exclude studies that compare TEAS with any other complementary and alternative therapies.

\section{Types of outcome measures}

\section{Primary outcomes}

The primary outcomes include changes in the revised Piper fatigue scale. ${ }^{26}$ It is a well-recognised and commonly used multidimensional measure in the CRF research field and contains 22 items and four subscales with a total score of 10 , and each score section represents the corresponding severity of fatigue ( 0 for none, 1-3 for mild, 4-6 for moderate and 7-10 for severe fatigue). CRF scores measured with other tools will also be included such as the brief fatigue inventory, the multidimensional fatigue inventory and the functional assessment of chronic illness therapy-fatigue.

\section{Secondary outcomes}

The secondary outcomes will include the quality-of-life measurement index, the anxiety and depression levels measured by qualified scales such as the Hamilton anxiety scale and the Hamilton depression scale, and adverse events.

Patients and public involvement

No patient involved.

\section{Search methods for identification of studies}

\section{Electronic searches}

Two reviewers (YZ, JX) will independently search Cochrane Central Register of Controlled Trials (CENTRAL), PubMed, Embase, China National Knowledge Infrastructure, VIP, Wanfang database, Chinese Biomedical Literature Database, ClinicalTrials.gov ( www.clinicaltrials.gov) and WHO International Clinical Trial Registry Platform (www.who.it.trialsearch) from inception to 31 January 2021. The lists of references of retrieved articles will be searched for identifying potentially eligible trials. Language restriction will not be imposed on the electronic searches. We will use the following terms in a combination for the search: fatigue, asthenia, cancer-related fatigue, CRF, cancer, carcinoma, 


\begin{tabular}{|c|c|}
\hline Search line & Search items \\
\hline$\# 1$ & fatigue [MeSH Terms] \\
\hline \#2 & fatigue \\
\hline \#3 & cancer-related fatigue \\
\hline \#4 & cancer \\
\hline \#5 & carcinoma \\
\hline \#6 & malignance \\
\hline$\# 7$ & \#1 OR \#2 OR \#3 OR \#4 OR \#5 OR \#6 \\
\hline \#8 & transcutaneous electrical acupoint stimulation \\
\hline$\# 9$ & transcutaneous acupoint electrical stimulation \\
\hline$\# 10$ & TEAS \\
\hline$\# 11$ & $\begin{array}{l}\text { transcutaneous electrical acupuncture } \\
\text { stimulation }\end{array}$ \\
\hline$\# 12$ & $\begin{array}{l}\text { transcutaneous electrical acupuncture point } \\
\text { stimulation }\end{array}$ \\
\hline \#13 & \#8 OR \#9 OR \#10 OR \#11 OR \#12 \\
\hline \#14 & \#7 AND \#13 \\
\hline \#15 & Clinical trials [MeSH Terms] \\
\hline \#16 & Randomized [Title/Abstract] \\
\hline$\# 17$ & Randomly [Title/Abstract] \\
\hline$\# 18$ & Trial [Title] \\
\hline \#19 & \#15 OR \#16 OR \#17 OR \#18 \\
\hline \#20 & \#7 AND \#14 AND \#19 \\
\hline
\end{tabular}

TEAS, transcutaneous electrical acupoint stimulation.

tumor, malignancy, Transcutaneous electrical acupoints stimulation and TEAS. The search strategy for PubMed is shown in table 1 .

\section{Searching other sources}

The two reviewers will check the reference lists of all included articles to retrieve additional trials. Manual searching will be applied when abstracts are not available online. Unpublished literature will be searched via conference proceedings. Information unavailable in the articles will be acquired by contacting the authors when it is possible.

\section{Data collection and analysis}

Data extraction will be performed in accordance with the Cochrane Handbook for Systematic Reviews of Interventions, V.6.1. ${ }^{27}$ Data analysis will be conducted using RevMan V.5.4.1 (Review Manager 2020).

\section{Selection of studies}

Two reviewers (YZ, JX) will independently perform abstract screening. RCTs evaluating the efficacy of TEAS for patients with CRF will be included. We will retrieve the full texts of all remaining articles and independently screen all the full-text articles according to the inclusion criteria. Disagreements will be settled by discussion, and when an agreement cannot be reached, the third review author (YR) will make the final
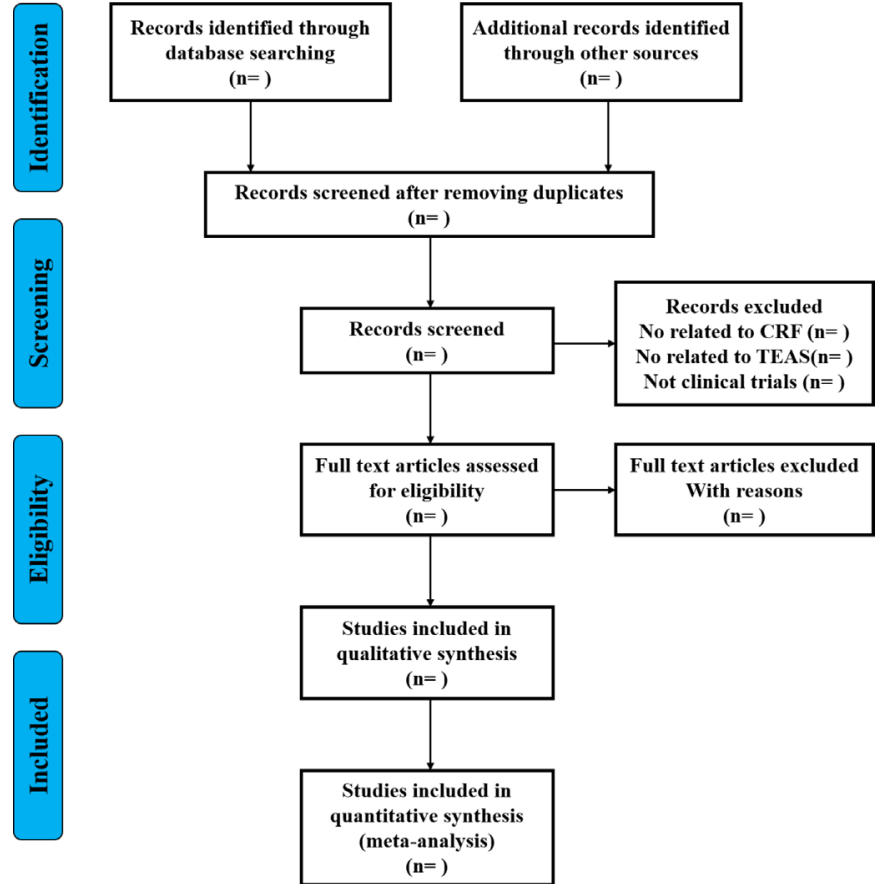

Figure 1 Flow diagram of the study selection process. CRF, cancer-related fatigue; TEAS, transcutaneous electrical acupoint stimulation.

decision. The PRISMA flow diagram of the selection process is presented in figure 1 .

\section{Data extraction and management}

We will design a data extraction form and two authors (YZ, JX) will independently extract the data from the eligible studies. It includes the following information: reference ID, author, year of publication, participant characteristics (eg, age, gender, duration and severity of the disease), sample size, randomization method, allocation concealment method, blinding method, interventions, analytic set, number of participants analysed, outcome measures, adverse events, and follow-up. Discrepancies will be resolved through discussion or by the third reviewer (YR). For unclear information, we will contact the first or corresponding author.

\section{Assessment of risk of bias in included studies}

Two reviewers will independently assess the risk of bias for each RCTs included using Cochrane Collaboration's risk of bias tool, which contains seven domains: random sequence generation, allocation concealment, blinding of participants and personnel, blinding of outcome assessment, incomplete outcome data, selective reporting, and other sources of bias. In each domain, the risk of bias will be classified as 'low risk' of bias, 'high risk' of bias or 'unclear risk' of bias. ${ }^{28}$ Disagreement will be resolved by discussion or consensus with the third author.

\section{Data analysis}

Data synthesis

The process will be performed using RevMan V.5.4.1 (Review Manager 2020). Trials that have the same 
outcome measures in similar populations will be combined to estimate the pooled effect. For dichotomous data, a risk ratio (RR) with $95 \%$ CIs will be used as pooled statistics. For numeric variables, standardised mean difference (SMD) with $95 \%$ CIs will be used considering that the primary outcome is the scoring scale. If the $\mathrm{RR}$ or SMD is not available, we will try to recalculate them using the reported data, including the median, $p$ values and CIs. The hypothesis test will apply the inverse variance method for numeric data and the Mantel-Haenszel method for dichotomous data. A $p$ value less than 0.05 $(\mathrm{p}<0.05)$ is statistically significant. If the meta-analysis is unfeasible, we will provide a narrative description of the results.

\section{Assessment of heterogeneity}

Heterogeneity will be assessed using the $\chi^{2}$ test and $\mathrm{I}^{2}$ statistics. When there is no significant heterogeneity $\left(\mathrm{I}^{2}<50 \%\right.$ and $\left.\mathrm{p}>0.1\right)$, the fixed-effect model will be applied. The random-effects model will be applied for significant heterogeneity $\left(80 \%>\mathrm{I}^{2}>50 \%\right.$ and $\left.\mathrm{p}<0.1\right)$.

\section{Subgroup analysis and sensitivity analysis}

Subgroup analysis will be performed based on the primary and secondary outcome measures to detect possible causes of heterogeneity. The following subgroups will be investigated respectively: different types of the control (eg, wait-list, TEAS on non-acupoints, acupuncture and drugs), treatment duration and the severity of symptoms at baseline. A sensitivity analysis will be performed to evaluate the robustness and stability of the evidence, analyses will be limited to studies with a low risk of bias, and one study will be iteratively removed at a time.

\section{Assessment of publication bias}

Publication bias will be analysed using funnel plots if there are more than ten studies included. A symmetrically distributed funnel plot indicates that there is no publication bias. If less than ten articles are included, Egger and Begg tests will be applied.

\section{Summary of evidence}

Grading of Recommendations Assessment, Development and Evaluation will be applied to classify the quality of evidence as 'high', 'moderate', 'low' and 'very low' in the domain of the risk of bias (methodological quality), indirectness of evidence, heterogeneity, precision of effect and publication bias in each study.

\section{Ethics and dissemination}

The results of this systematic review and mate-analysis will be disseminated in a peer-reviewed journal. No ethical approval is required since this study will not contain any private information of participants.

\section{Twitter Yulan Ren @renxg771211}

Contributors $\mathrm{YZ}, \mathrm{JX}, \mathrm{XT}$ and $\mathrm{YR}$ designed this study. XT and YR are the guarantors for the study. YZ and JX contributed equally to the drafting of the manuscript of this protocol, which is revised by YR and XT. All reviewers developed the research strategy. YZ and JX will independently carry out the search, selection and identification of studies and the data extraction. YZ and XT will perform the data synthesis and analysis. YR will be served as the third reviewer for settlement of disagreement. YR and ZC will be the adviser for methodology. All authors have approved the publication of this protocol.

Funding This study was supported by a major R\&D project of the Sichuan Provincial Department of Science and Technology of China (approval number: 2018SZ071).

Competing interests None declared.

Patient and public involvement Patients and/or the public were not involved in the design, or conduct, or reporting, or dissemination plans of this research.

Patient consent for publication Not applicable.

Provenance and peer review Not commissioned; externally peer reviewed.

Open access This is an open access article distributed in accordance with the Creative Commons Attribution Non Commercial (CC BY-NC 4.0) license, which permits others to distribute, remix, adapt, build upon this work non-commercially, and license their derivative works on different terms, provided the original work is properly cited, appropriate credit is given, any changes made indicated, and the use is non-commercial. See: http://creativecommons.org/licenses/by-nc/4.0/.

ORCID iDs

Yiwei Zeng http://orcid.org/0000-0002-7042-2503

Zhihan Chen http://orcid.org/0000-0002-0237-7501

\section{REFERENCES}

1 Berger AM, Abernethy AP, Atkinson A, et al. NCCN clinical practice guidelines cancer-related fatigue. J Natl Compr Canc Netw 2010;8:904-31.

2 Hofman M, Ryan JL, Figueroa-Moseley CD, et al. Cancer-related fatigue: the scale of the problem. Oncologist 2007;12:4-10.

3 Weis J. Cancer-related fatigue: prevalence, assessment and treatment strategies. Expert Rev Pharmacoecon Outcomes Res 2011;11:441-6.

4 Fabi A, Bhargava R, Fatigoni S, et al. Cancer-related fatigue: ESMO clinical practice guidelines for diagnosis and treatment. Ann Oncol 2020;31:713-23.

5 Thong MSY, van Noorden CJF, Steindorf K, et al. Cancer-related fatigue: causes and current treatment options. Curr Treat Options Oncol 2020;21:17.

6 Neefjes ECW, Vorst MJDL, Blauwhoff-Buskermolen S, et al. Aiming for a better understanding and management of cancer-related fatigue. Oncologist 2013;18:1135-43.

7 Gilliam LAA, St. Clair DK. Chemotherapy-induced weakness and fatigue in skeletal muscle: the role of oxidative stress. Antioxid Redox Signal 2011;15:2543-63.

8 Bower JE. Cancer-related fatigue-mechanisms, risk factors, and treatments. Nat Rev Clin Oncol 2014;11:597-609.

9 Barsevick A, Frost M, Zwinderman A, et al. I'm so tired: biological and genetic mechanisms of cancer-related fatigue. Qual Life Res 2010;19:1419-27.

10 Curt GA, Breitbart W, Cella D, et al. Impact of cancer-related fatigue on the lives of patients: new findings from the fatigue coalition. Oncologist 2000;5:353-60.

11 Santoni M, Conti A, Massari F, et al. Treatment-related fatigue with sorafenib, sunitinib and pazopanib in patients with advanced solid tumors: an up-to-date review and meta-analysis of clinical trials. Int J Cancer 2015;136:1-10.

12 Mitchell SA. Cancer-related fatigue: state of the science. Pm R 2010;2:364-83.

13 Mustian KM, Alfano CM, Heckler C, et al. Comparison of pharmaceutical, psychological, and exercise treatments for cancerrelated fatigue. JAMA Oncol 2017;3:961-8.

14 Koornstra RHT, Peters M, Donofrio S, et al. Management of fatigue in patients with cancer - a practical overview. Cancer Treat Rev 2014;40:791-9.

15 Xiang Y, Guo Z, Zhu P, et al. Traditional Chinese medicine as a cancer treatment: modern perspectives of ancient but advanced science. Cancer Med 2019;8:1958-75.

16 Oh B, Eade T, Kneebone A, et al. Factors affecting whether or not cancer patients consider using acupuncture. Acupunct Med 2017;35:107-13.

17 Zhang Y, Lin L, Li H, et al. Effects of acupuncture on cancer-related fatigue: a meta-analysis. Support Care Cancer 2018;26:415-25. 
18 Tian Z-X, Liu C-Z, Qi Y-S, et al. Transcutaneous electrical acupoint stimulation for stage 1 hypertension: protocol for a randomized controlled pilot trial. Trials 2020;21:558.

19 Hou L, Zhou C, Wu Y, et al. Transcutaneous electrical acupoint stimulation (TEAS) relieved cancer-related fatigue in non-small cell lung cancer (NSCLC) patients after chemotherapy. $J$ Thorac Dis 2017:9:1959-66.

20 Tu Q, Yang Z, Gan J, et al. Transcutaneous electrical Acupoint stimulation improves immunological function during the perioperative period in patients with non-small cell lung cancer undergoing videoassisted thoracic surgical lobectomy. Technol Cancer Res Treat 2018; $17: 153303381880647$

21 Hou L, Gu F, Gao G, et al. Transcutaneous electrical acupoint stimulation (TEAS) ameliorates chemotherapy-induced bone marrow suppression in lung cancer patients. J Thorac Dis 2017:9:809-17.

22 Jiang Y, Wang H, Liu Z, et al. Manipulation of and sustained effects on the human brain induced by different modalities of acupuncture: an fMRI study. PLoS One 2013;8:e66815.

23 Feng B, Zhang Y, Luo L-Y, et al. Transcutaneous electrical acupoint stimulation for post-traumatic stress disorder: assessor- blinded, randomized controlled study. Psychiatry Clin Neurosci 2019;73:179-86.

24 Preferred reporting items for systematic review and meta-analysis protocols (PRISMA-P) 2015: elaboration and explanation. BMJ 2016;354:i4086.

25 Moher D, Shamseer L, Clarke M, et al. Preferred reporting items for systematic review and meta-analysis protocols (PRISMA-P) 2015 statement. Syst Rev 2015;4.

26 Piper BF, Dibble SL, Dodd MJ, et al. The revised Piper fatigue scale: psychometric evaluation in women with breast cancer. Oncol Nurs Forum 1998;25:677-84.

27 Higgins JPT, Thomas J, Chandler J. Cochrane Handbook for systematic reviews of interventions version 6.1 (updated September 2020). Cochrane, 2020. Available: www.training.cochrane.org/ handbook

28 Higgins JPT, Altman DG, Gøtzsche PC, et al. The Cochrane collaboration's tool for assessing risk of bias in randomised trials. BMJ 2011;343:d5928. 